\title{
The influence of bacteria on the passive film stability of 304 stainless steel
}

\author{
Kh.M. Ismail ${ }^{1}$, A. Jayaraman ${ }^{2}$, T.K. Wood $^{3}$, J.C. Earthman * \\ Department of Chemical and Biochemical Engineering and Materials Science, University of California, 916 Engineering Tower, \\ Irvine, CA 92697-2575, USA
}

Received 7 December 1998; received in revised form 15 April 1999

\begin{abstract}
The stability of stainless steel 304 in the presence of aerobic and anaerobic bacteria was investigated in a continuous flow system using electrochemical impedance spectroscopy and scanning electron microscopy (SEM) examinations. The results show that the open circuit potential (OCP) of stainless steel was ennobled (shifted in the electropositive direction) by about $+150 \mathrm{mV}$ in the presence of the aerobic bacteria. The observed change of OCP in the electropositive direction can be explained by an increase in the rate of the cathodic reaction. Also the presence of an aerobic biofilm led to a decrease in the polarization resistance of stainless steel is not only due to the growth of micropits, as shown from SEM micrographs, but also due to thinning of the passive film. In the presence of only Pseudomonas fragi, the electrochemical impedance response showed a capacitive behavior with RP on the order of 500 $\mathrm{k} \Omega$. The addition of anaerobic sulfate reducing bacteria (SRB) to the test medium decreased RP to $12 \mathrm{k} \Omega$ due to an increase in localized corrosion, as indicated by SEM examination. (C) 1999 Elsevier Science Ltd. All rights reserved.
\end{abstract}

Keywords: Biofilm; Aerobic/anaerobic bacteria; EIS; SEM; Stainless steel 304; Pseudomonas fragi; Sulfate reducing bacteria (SRB); Desulfovibrio vulgaris

\section{Introduction}

Type 304 stainless steel has been used increasingly for cooling water service in the chemical, petrochemical and power utility industries. This has often been done to eliminate the need for corrosion inhibitors, with their environmental concerns. The excellent corrosion resistance of stainless steel is due to the formation of a

\footnotetext{
* Corresponding author. Tel.: + 1-949-8245018; fax: + 1949-8243812.

E-mail address: earthman@uci.edu (J.C. Earthman)

${ }^{1}$ Present address: Department of Chemistry, University of Cairo, Eqypt.

${ }^{2}$ Present address: Center for Engineering in Medicine, Massachussetts General Hospital, Cambridge, MA 02139, USA.

${ }^{3}$ Present address: Department of Chemical Engineering, University of Connecticut, Storrs, CT 06269, USA.
}

stable passive layer. Nevertheless, stainless steel is susceptible to localized corrosion by chloride ions and reduced sulfur compounds [1].

The presence of microorganisms on a metal surface often leads to highly localized changes in the concentration of the electrolyte constituents, $\mathrm{pH}$ and oxygen levels [2-4]. These microorganisms and their metabolic activity were clearly believed to influence the corrosion process [5]. Microorganisms often stimulate localized forms of corrosion, such as pitting, depending on the passive film forming and repairing capabilities of the metal or alloy [6].

The present study is designed to gain better understanding of the influence of aerobic and anaerobic bacteria on the stability of stainless steel using electrochemical impedance spectroscopy (EIS) and scanning electron microscopy (SEM) techniques. EIS was used to 
investigate the electrochemical behavior and to measure the polarization resistance of the stainless steel. The open circuit potential (OCP) was also monitored to detect the bacterial biofilm activities such as oxygen depletion and other electrochemical changes.

\section{Experimental methods}

\subsection{Test reactors}

The test reactors were designed to support continuous bacterial cultures and allow the taking of $\mathrm{AC}$ and DC electrochemical readings. Fig. 1 shows the reactor configuration that allows continuous flow of nutrients medium and air, and is equipped with a counter electrode and a reference electrode for electrochemical measurements. Air enters the reactor at a point just above the level of the medium to provide oxygen to the solution. The counter electrode is made of titanium to resist corrosion in different environments and is particularly resistant to microbiologically influenced corrosion (MIC). An autoclavable silver/silver chloride electrode was used as a reference electrode. A metal specimen under investigation is placed at the bottom of the glass cylinder, leaving $26.4 \mathrm{~cm}^{2}$ in contact with the solution, and Teflon ${ }^{\circledR}$ plates were used above and below the cylinder to provide electrical insulation.

\subsection{Medium}

All tests were conducted using a nutrient-rich modified Baar's medium. The modified Baar's medium consists of $4.12 \mathrm{~g} \mathrm{MgSO}_{4} \cdot 7 \mathrm{H}_{2} \mathrm{O}, 5 \mathrm{~g}$ sodium citrate, $1.26 \mathrm{~g} \mathrm{CaSO}_{4} \cdot 2 \mathrm{H}_{2} \mathrm{O}, 1.0 \mathrm{~g} \mathrm{NH}_{4} \mathrm{Cl}, 1.0 \mathrm{~g} \mathrm{~K}_{2} \mathrm{HPO}_{4}, 3.5 \mathrm{~g}$ sodium lactate, and $1.0 \mathrm{~g}$ yeast extract. The $\mathrm{pH}$ of the medium was adjusted to 7.2 which is assumed not to change during the experiments due to the continuous flow of the medium. Sealed 2-1 flasks containing 1.51 of nutrient medium were autoclaved at $121^{\circ} \mathrm{C}$ for $20 \mathrm{~min}$ and stored at room temperature until use.

\subsection{Specimen preparation}

The samples were machined from type 304 stainless steel sheet with dimensions $7.64 \times 7.64 \times 0.1 \mathrm{~cm}$. The stainless steel specimens were then polished to 240 grit sandpaper. Then the samples were rinsed in distilled water, air dried, and stored until use.

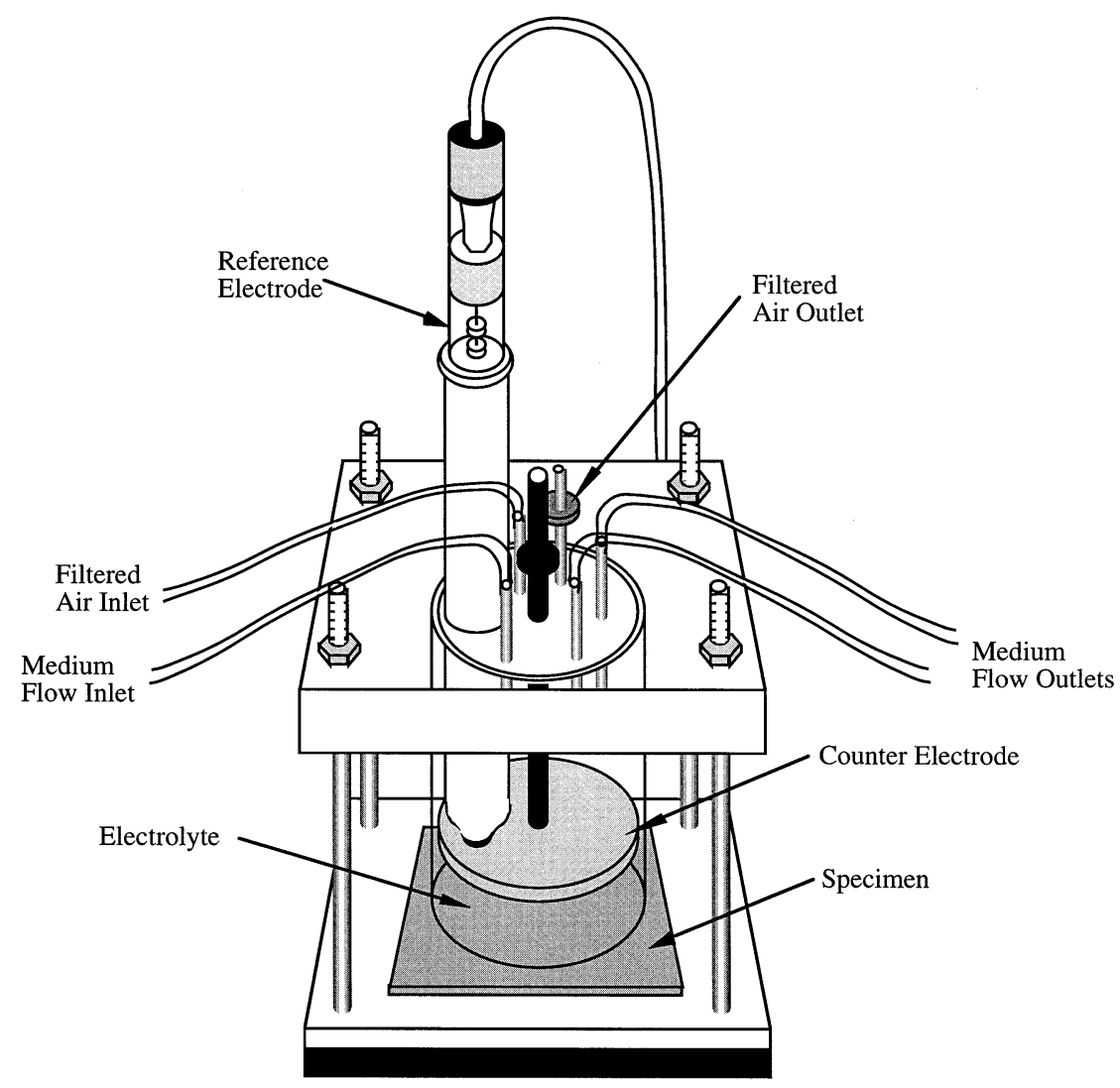

Fig. 1. Schematic representation of the continuous flow reactor used for electrochemical measurements. 


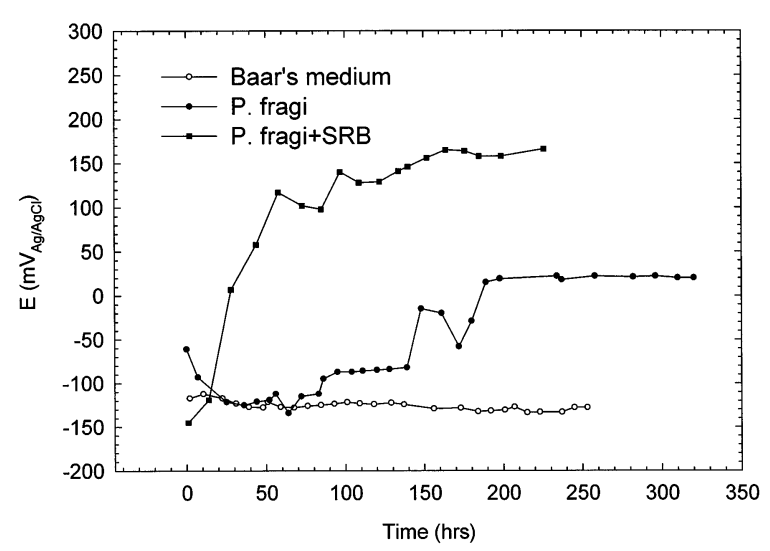

Fig. 2. Open circuit potential for stainless steel in sterile Baar's, in the presence of $P$. fragi, and in the presence of $P$. fragi and $\mathrm{SRB}$ at $30^{\circ} \mathrm{C}$.

\subsection{Bacterial culture}

Pseudomonas fragi were obtained from the American Type Culture Collection (ATCC no. 4973). A Kanamycin (antibiotic) resistant derivative of the strain was then obtained to avoid contamination (providing assurance that a mono-culture will be maintained since P. fragi will be able to survive the Kanamycin in the tested media). The bacteria were cultivated overnight in $250 \mathrm{ml}$ Erlenmeyer flasks containing $10 \mathrm{ml}$ of LB medium in a rotary shaker $\left(250 \mathrm{rpm}, 30^{\circ} \mathrm{C}\right)$. A total of $1 \mathrm{ml}$ of the cultured bacteria was used to inoculate each of the test reactors.

\subsection{Dual culture experiments}

Anaerobic sulfate reducing bacteria (SRB), Desulfovibrio vulgaris, were added in some experiments to already growing aerobic bacteria to examine the combined effect of the aerobic bacteria with that for SRB. We observed that SRB can be active in an anaerobic bottom layer when the bulk solution contains oxygen. Formation and maintenance of such anaerobic conditions are due to the presence of aerobic bacteria. The respiration of aerobic bacteria scavenges oxygen and favors growth conditions for SRB. In these experiments, the SRB were added from 3 to 7 days after the inoculation of the medium with the aerobic bacteria. Hence, a biofilm produced by the aerobic bacteria was present on the samples prior to the addition of SRB. The stability of the specimen in the presence of the dual culture was then monitored on a regular basis using EIS techniques.

\subsection{Electrochemical measurements}

Electrochemical impedance spectroscopy (EIS) measurements were performed using a Solartron-Schlumberger electrochemical measurement unit (SI 1280) connected to a desktop computer. The program that controls the SI 1280 and records the readings was developed in-house using the Labview graphical programming language. Measurements were made at the open circuit potential using a $10 \mathrm{mV}$ amplitude sinusoidal signal over frequencies ranging from 20000 to $0.001 \mathrm{~Hz}$. EIS readings were taken every $6 \mathrm{~h}$. The duration of a run varied between the tests, ranged from 10 to 40 days.

\subsection{Scanning electron microscopy}

Morphology and magnitude of the corrosion protection were observed using a Philips XL30 scanning electron microscope. The impedance measurements were recorded until $R_{\mathrm{P}}$ reached a steady state value, then the metal specimens were removed, cleaned, dried, and examined using SEM.

\subsection{Testing procedures}

The entire reactor apparatus including the reference electrode is autoclavable; the media and reactors were autoclaved at $121^{\circ} \mathrm{C}$ for $20 \mathrm{~min}$ prior to testing. An experiment with a sterile control reactor was performed in tandem for each bacterial reactor tested. A continuous flow reactor, containing $100 \mathrm{ml}$ of medium, was inoculated with $1 \mathrm{ml}$ of the cultured bacteria. Continuous flow systems were used in this study because they are more characteristic of the service water systems of interest than are batch culture systems. However, during the initial $12 \mathrm{~h}$ the reactor was held in batch mode to allow colonization of the metal surface. Subsequently, the reactor was switched to continuous flow with a solution flow rate $12 \mathrm{ml} \mathrm{h}^{-1}$ except when otherwise stated. Baar's medium flowed through both the sterile control reactors and the reactors inoculated with $P$. fragi. Air was flowed at the top of the medium at a rate of $100 \mathrm{ml} \mathrm{min}^{-1}$ to provide an ample supply of oxygen over the duration of the experiment. EIS measurements were carried out at the open circuit potential (OCP) until steady state was achieved. Each experiment was discontinued once the readings stabilized. All measurements were carried out at $30^{\circ} \mathrm{C}$ for optimum bacteria growth.

\section{Results and discussion}

Each test was allowed to run until the open circuit potential $(\mathrm{OCP})$ and the polarization resistance, $R_{\mathrm{P}}$, 
reached a steady state value. Fig. 2 shows the variations of the OCP with time for stainless steel in Baar's medium at $30^{\circ} \mathrm{C}$. In sterile medium, no significant changes in OCP occur. In the presence of only P. fragi, the OCP of stainless steel was ennobled (shifted in the electropositive direction) by about $+150 \mathrm{mV}$ (from -130 to $+20 \mathrm{mV} \mathrm{Ag} / \mathrm{AgCl})$. The addition of $P$. fragi and SRB shifted OCP values at a rate faster than that observed in the presence of $P$. fragi alone. Also, the addition of SRB leads to further shift of the OCP by about $+150 \mathrm{mV}$ (to about $+170 \mathrm{mV} \mathrm{Ag} / \mathrm{AgCl}$ ). This ennoblement of the OCP with the addition of $P$. fragi

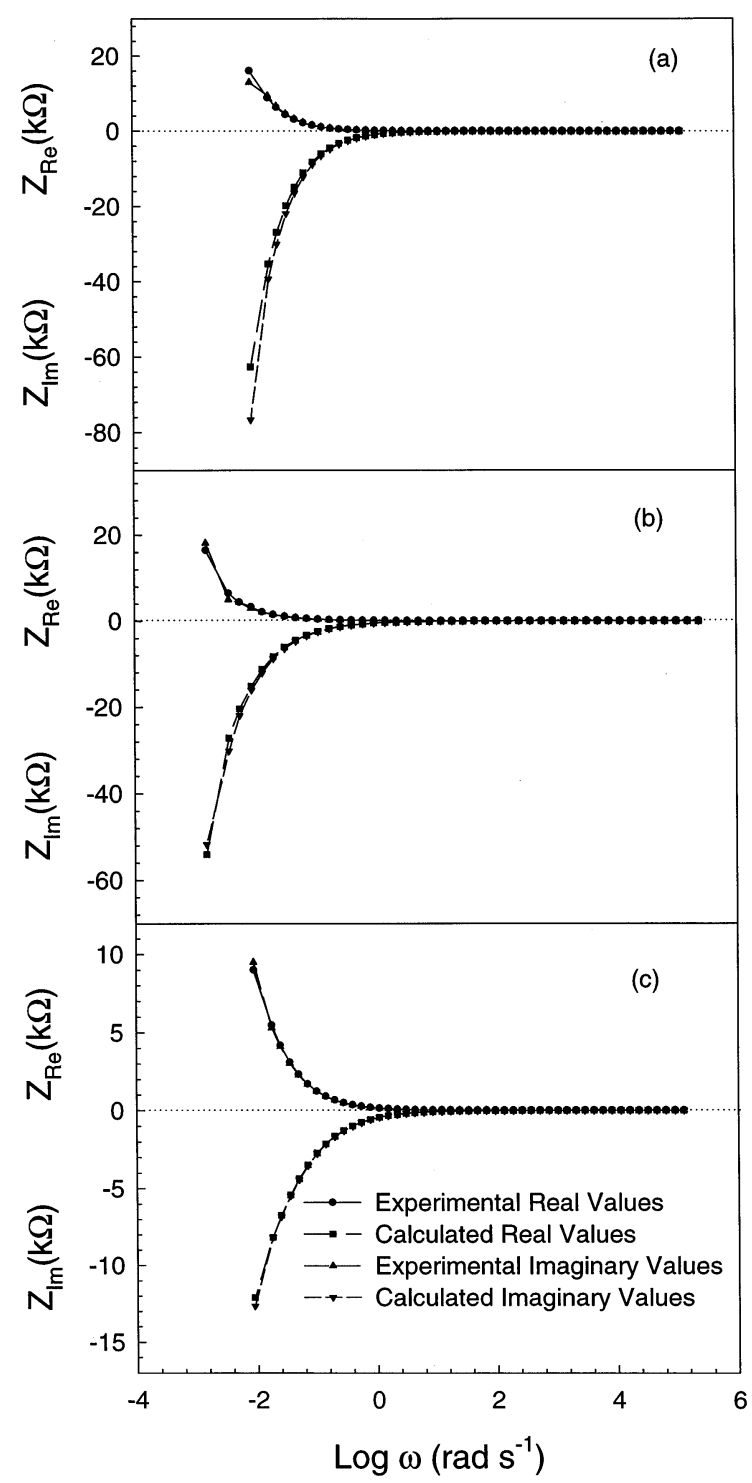

Fig. 3. $\mathrm{K}-\mathrm{K}$ transforms of real and imaginary components of the EIS data for stainless steel in Baar's medium at OCP (a) sterile; (b) P. fragi; (c) P. fragi + SRB.

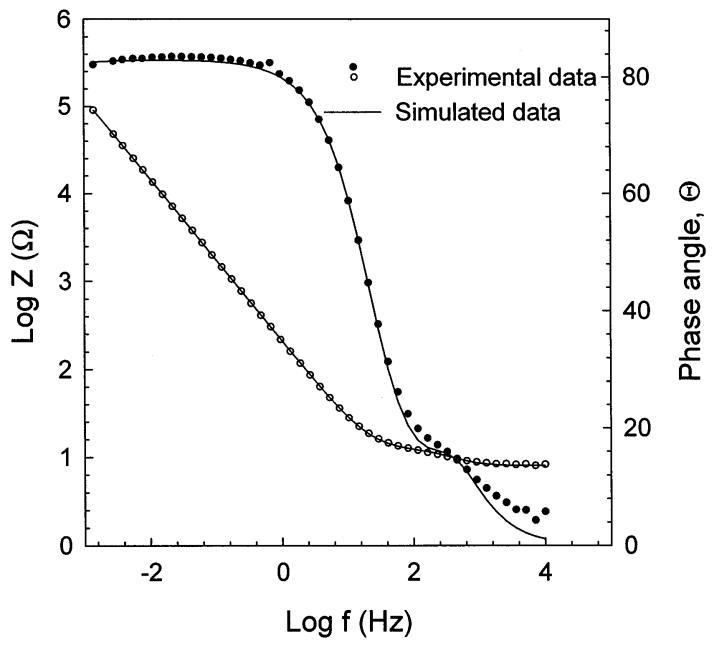

Experimental data

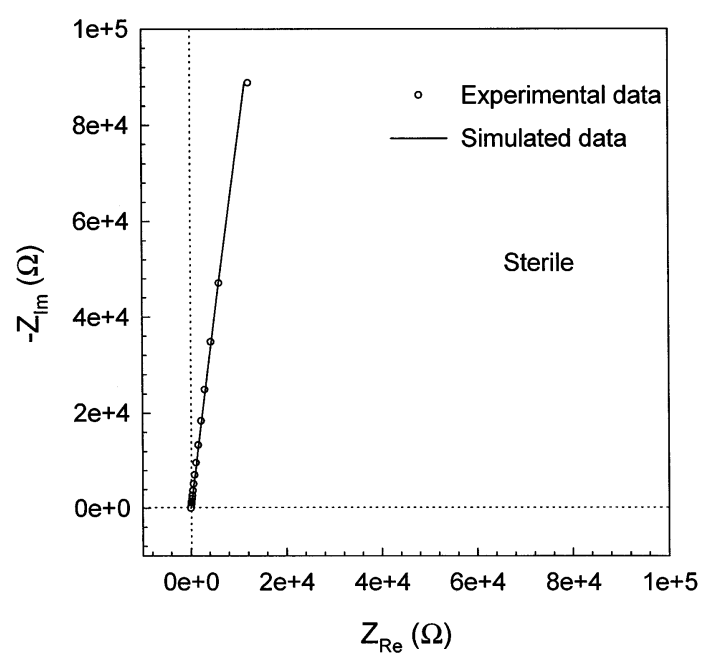

Fig. 4. Electrochemical impedance spectra for stainless steel after $183 \mathrm{~h}$ in sterile Baar's medium, at $30^{\circ} \mathrm{C}$, solution flow rate $12 \mathrm{ml} \mathrm{h}^{-1}$, at OCP: (a) Bode plot; (b) Nyquist plot.

and SRB could be due to a positive shift of the reversible potential of the cathodic reaction as a result of a significant decrease of $\mathrm{pH}$ under biofilms [3].

Simple inspection of the electrochemical impedance data does not necessarily reveal whether or not the data are valid or have been distorted by some experimental artifact. However the validity of the data can be assessed using the Kramers-Kronig $(\mathrm{K}-\mathrm{K})$ transforms. Any system that satisfies the conditions of linearity, stability, and causality a priori must satisfy the $\mathrm{K}-\mathrm{K}$ transforms $[7,8]$. The $\mathrm{K}-\mathrm{K}$ technique transforms the real component into the imaginary component and vice versa, so that the transformed quantities may be compared directly with experimental values for the same 

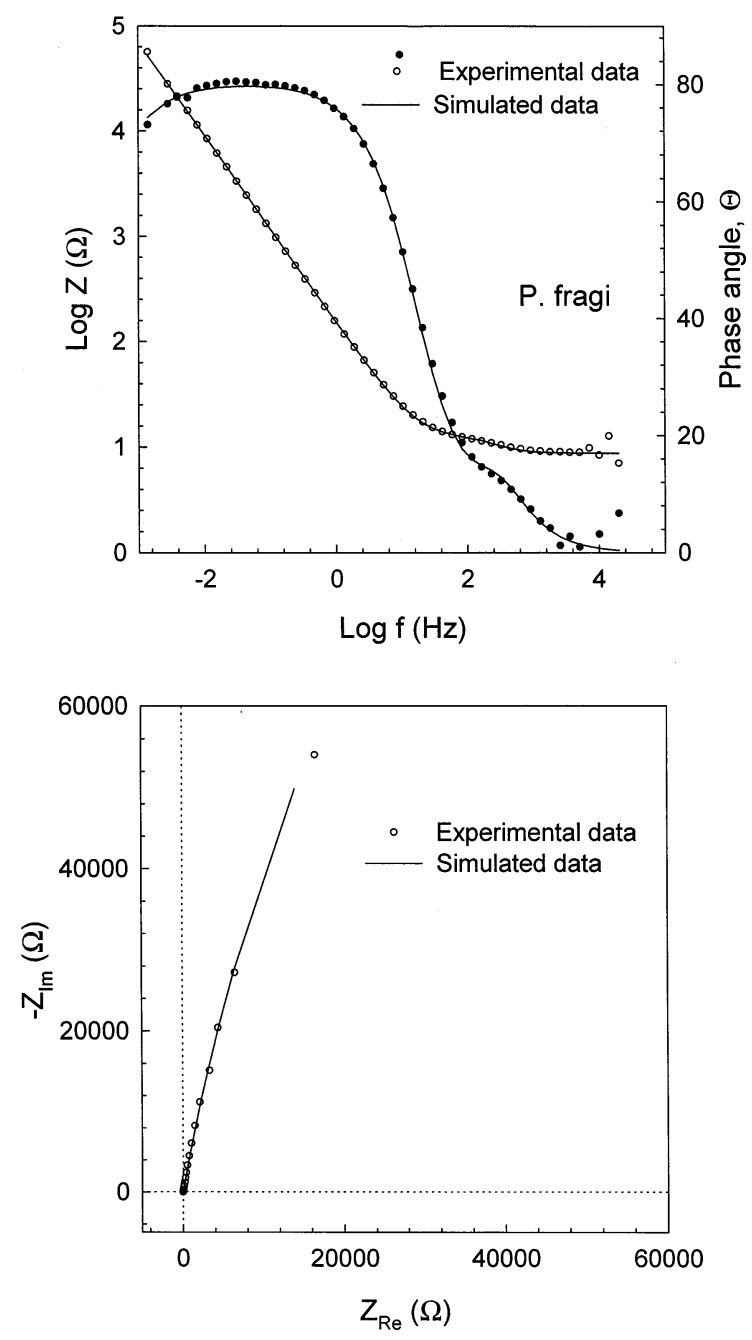

Fig. 5. Electrochemical impedance spectra for stainless steel after $139 \mathrm{~h}$ in the presence of $P$. fragi at $30^{\circ} \mathrm{C}$, solution flow rate $12 \mathrm{ml} \mathrm{h}^{-1}$, at OCP: (a) Bode plot; (b) Nyquist plot.

parameters. As can be seen from Fig. 3, the high fidelity between the experimental and transformed impedance data for both real and imaginary components show that the system under investigation comply with the linearity, causality, and stability constraints of linear system theory and thereby validates the EIS data.

Impedance spectra for stainless steel samples in the sterile Baar's medium remained virtually unchanged with exposure time. An example of these spectra for an exposure period of $183 \mathrm{~h}$ is shown in Fig. 4. Impedance data for all control samples showed capacitive behavior with $R_{\mathrm{P}}$ exceeding $12 \mathrm{M} \Omega$ (calculated using equivalent circuit), and remaining practically unchanged with exposure time, indicating that localized corrosion did not occur. No signs of localized corrosion were detected visually, microscopically or electrochemically over the entire experiment period. SEM micrographs provide further evidence that localized corrosion did not occur under sterile control conditions.

Typical EIS data for stainless steel in Baar's medium inoculated with $P$. fragi bacteria are illustrated in Fig. 5. Aerobic biofilm formation in Baar's medium on several samples of stainless steel at OCP produces an apparent decrease in the polarization resistance, $R_{\mathrm{P}}$, in comparison with those obtained in the sterile control experiments. In essence, EIS spectra remained unchanged over time, indicating capacitive behavior and a slightly lower $R_{\mathrm{P}}$ in comparison with the sterile control. This decline in EIS could be attributed to thinning of the passive film due to the presence of aerobic bacteria. Activity of slime forming bacteria reduces dissolved
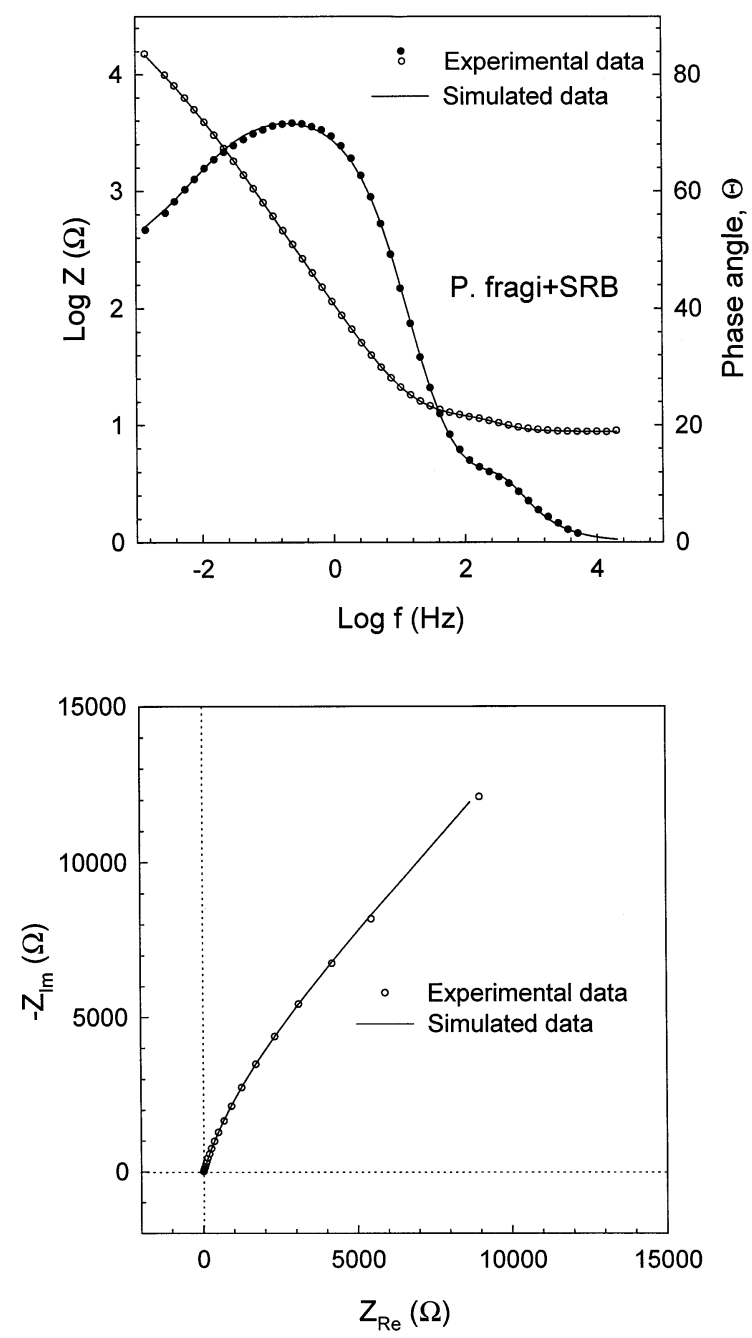

Fig. 6. Electrochemical impedance spectra for stainless steel after $903 \mathrm{~h}$ in the presence of $P$. fragi $+\mathrm{SRB}$ at $30^{\circ} \mathrm{C}$, solution flow rate $12 \mathrm{ml} \mathrm{h}^{-1}$, at OCP: (a) Bode plot; (b) Nyquist plot. 


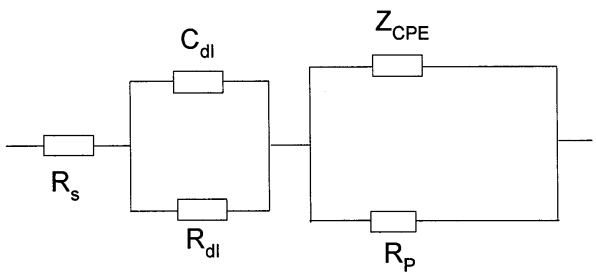

(a)

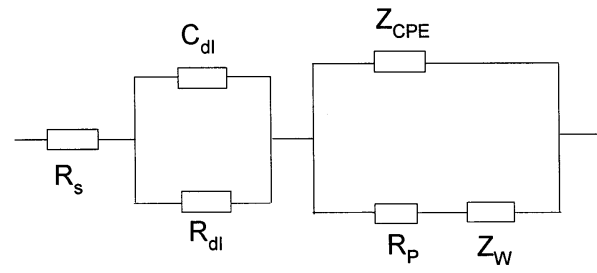

(b)

Fig. 7. Two equivalent circuits used in fitting impedance data of stainless steel at different conditions.

oxygen concentration and restricts access of fresh oxygen to the metal surface. This reduces the formation of fresh oxide by reaction of the metal with dissolved oxygen. Nevertheless, the formation of micropits in the presence of heterogeneous biofilm distribution can not be eliminated.

The electrochemical impedance spectra obtained from stainless steel in the presence of $P$. fragi and SRB suggest a greater reduction in $R_{\mathrm{P}}$ in comparison with those obtained in the sterile control experiments (Fig. 6 ). The alteration of the oxide layer structure facilitates the formation of localized corrosion when another aggressive anion, such as chloride, is present [9]. Furthermore, reduced sulfur compounds in the complete absence of chloride can induce pitting corrosion of stainless steel [1]. Also it is well known that deposits can enhance localized corrosion of stainless steel. The corrosion reaction at an induced anodic site under a deposit would be supported by a cathodic reaction at the metal sulfides deposits and significant localized corrosion initiates. The formation of an imperfect oxide layer on stainless steel in the presence of sulfur has been reported [10]. Thus, it is reasonable to assume that the growth of SRB not only reduces the passive film thickness by forming metal sulfides but also initiates localized corrosion.

The impedance spectra obtained experimentally were analyzed using equivalent circuit software [11] and two equivalent circuits from which the parameters were calculated. According to AC circuit theory, an impedance plot obtained for a given electrochemical system can be correlated with one or more equivalent circuits. Sometimes simple elements such as capacitors, resistors, and diffusion-related elements are not sufficient to represent a measured impedance [12]. Data for stainless steel in sterile medium or in the presence of $P$. fragi were analyzed using the equivalent circuit illustrated in Fig. 7a. As shown in this figure, the passive film is represented by a constant phase element impedance, $Z_{\mathrm{CPE}}$ in parallel with passive film resistance.
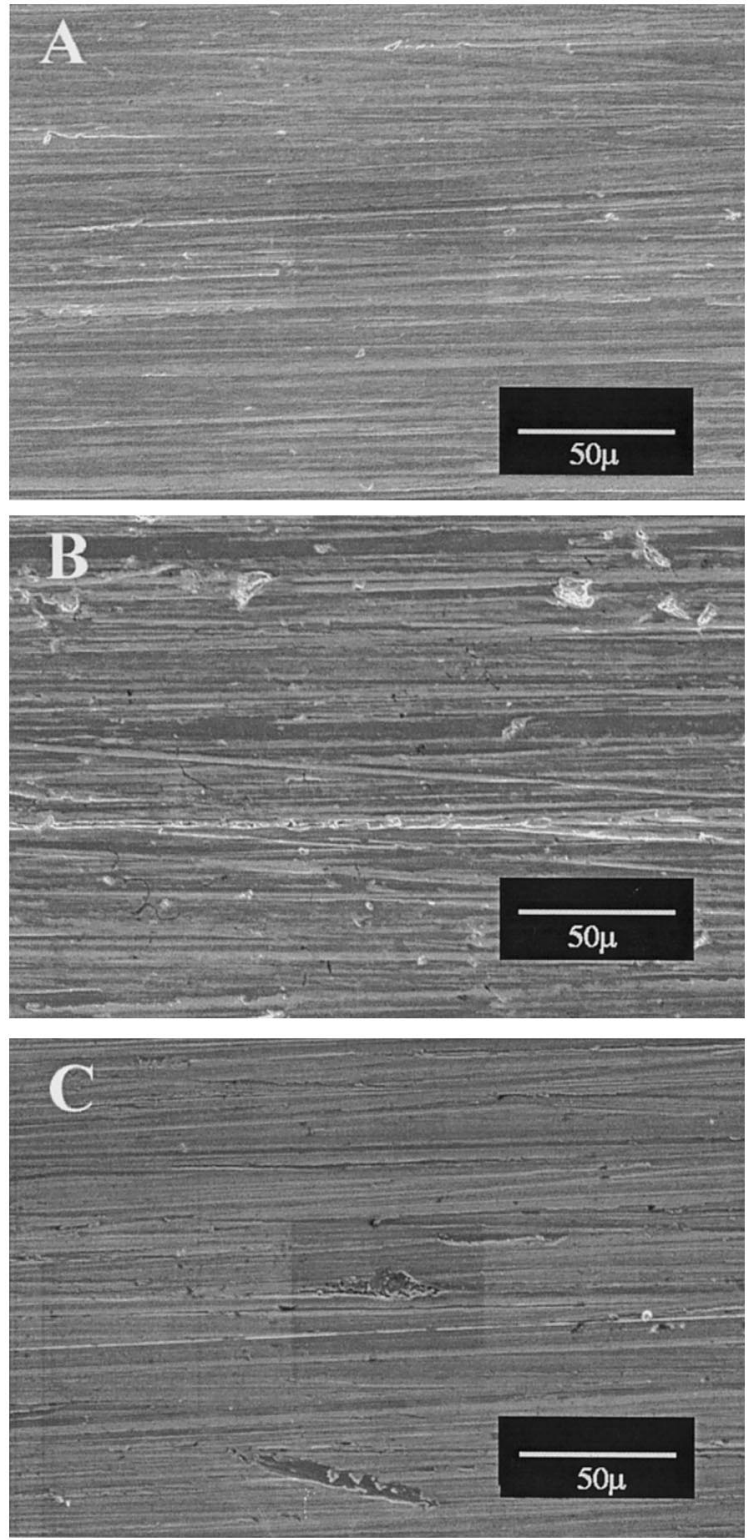

Fig. 8. (A) SEM micrographs of stainless steel exposed to aerated Baar's medium sterile control for 8 days; (B) in the presence of $P$. fragi for 8 days; and (C) in the presence of $P$. fragi $+\mathrm{SRB}$ for 38 days at $30^{\circ} \mathrm{C}$. Low magnification $(525 \times)$. 
Table 1

Impedance parameters of stainless steel in Baar's medium at $30^{\circ} \mathrm{C}$, solution flow rate $12 \mathrm{ml} \mathrm{h}^{-1}$, at $\mathrm{OCP}$

\begin{tabular}{|c|c|c|c|c|c|c|c|}
\hline Parameters & $R_{\mathrm{s}}(\Omega)$ & $C_{\mathrm{dl}}\left(\mu \mathrm{Fcm}^{-2}\right)$ & $R_{\mathrm{dl}}(\Omega)$ & $Y\left(\Omega^{-1}\right)$ & $n$ & $R_{\mathrm{P}}(\mathrm{M} \Omega)$ & $W\left(\Omega^{-1}\right)$ \\
\hline Sterile & 8.1 & 5.3 & 3.5 & $8.8 \times 10^{-4}$ & 0.92 & 12.0 & - \\
\hline P. fragi & 8.8 & 8.3 & 3.0 & $1.3 \times 10^{-3}$ & 0.89 & 0.501 & - \\
\hline P. fragi $+\mathrm{SRB}$ & 8.8 & 8.0 & 2.5 & $2.0 \times 10^{-3}$ & 0.83 & 0.012 & $5.2 \times 10^{-4}$ \\
\hline
\end{tabular}

It can be seen for both sterile and inoculated medium the AC impedance response shows no diffusional effect. The $Z_{\mathrm{CPE}}$ impedance is described as: $Z_{\mathrm{CPE}}=1 / Y(j \omega)^{n}$ where $\omega$ is the angular frequency in $\operatorname{rad~s}^{-1}, Y$ is a constant phase element and $\mathrm{n}$ is an empirical exponent which can vary between 1 for a perfect capacitor, and 0 for a perfect resistor. A value of $n$ less than one would represent a somewhat imperfect capacitor and is generally thought to arise from the presence of heterogeneities both laterally and within the depth of the passive film [13]. The value of $n$ for stainless steel in sterile control medium was found to be less than one (Table 1) which reflects some degree of passive film heterogeneity. A lower value of $n$ was observed in the presence of $P$. fragi alone and in the presence of $P$. fragi and SRB. The heterogeneous distribution of the biofilms and the initiation of localized corrosion would expect to increase the passive film heterogeneity.

Data for stainless steel in the presence of $P$. fragi and SRB were analyzed using the equivalent circuit shown in Fig. $7 \mathrm{~b}$ where a Warburg impedance $\left(Z_{\mathrm{w}}\right)$ was introduced to account for a diffusion process within the metal sulfide layer. The associated double layer capacitance $\left(C_{\mathrm{dl}}\right)$ was found to be only on the order of several $\mu \mathrm{F} \mathrm{cm}{ }^{-2}$. This result suggests that $C_{\mathrm{dl}}$ is related to the Helmholtz double layer capacitance associated to the passive film/solution interface. Nevertheless, such a value of capacity is lower than the $C_{\mathrm{dl}}$, which is typically on the order of $20 \mu \mathrm{F} \mathrm{cm}^{-2}$.

Representative SEM micrographs are presented in Fig. 8 of stainless steel specimens that were exposed to an aerated Baar's medium (A) that was a sterile control for 8 days; (B) that contained $P$. fragi for 8 days; and (C) that contained P. fragi and SRB for 38 days. In the case of stainless steel specimens in sterile Baar's medium, there was no localized corrosion observed on the metal surface under open circuit conditions after 8 days (Fig. 8A). The presence of $P$. fragi in Baar's medium apparently initiates localized corrosion of the exposed specimens as indicated by the presence of micropits as shown in Fig. 8B. Somewhat larger pits were observed in the case of stainless steel specimens in the presence of $P$. fragi and SRB (Fig. 8C). Although localized corrosion in stainless steel can lead to large oscillations in corrosion potential values, the extent of the pitting in the presence of either $P$. fragi alone or $P$. fragi and SRB was not sufficient to cause large oscillations in the present OCP and EIS data.

\section{Conclusions}

- Electrochemical impedance spectroscopy and scanning electron microscopy were used to investigate the stability of stainless steel in the presence of an aerobic bacteria $P$. fragi, and a dual culture of $P$. fragi and an anaerobic sulfate reducing bacteria, $D$. vulgaris.

- Type 304 stainless steel shows no signs of pitting attack in the sterile Baar's medium.

- Stainless steel 304 is relatively stable in a sterile medium and in the presence of aerobic bacteria. However, corrosion micropits were observed for stainless steel in the presence of the aerobic bacteria. The combination of SRB and aerobic bacteria leads to a significant decrease in the value of $R_{\mathrm{P}}$ and gives rise to localized corrosion.

\section{Acknowledgements}

The authors gratefully acknowledge the support of this work by the Electric Power Research Institute (EPRI) under Contract No. WO8044-02.

\section{References}

[1] J.F.D. Stott, Corros. Sci. 35 (1993) 667.

[2] J.W. Costerton, Z. Lewandowski, D.E. Caldwell, D.R. Korber, H.M. Lappin-Scott, Ann. Rev. Microbiol. 49 (1995) 711

[3] S.C. Dexter, in: R. Baboian (Ed.), Corrosion Tests and Standards: Application and Interpretation, ASTM, Philadelphia, PA, 1995 (Chapter 43).

[4] S.M. Gerchakov, B.J. Little, P. Wagner, Corrosion 42 (1986) 689.

[5] V. Scotto, R. Di Cintio, G. Marcenaro, Corros. Sci. 25 (1985) 185

[6] S.C. Dexter, D.J. Duquette, O.W. Siebert, H.A. Videla, Corrosion 47 (4) (1991) 308.

[7] M. Urquidi-Macdonald, S. Real, D.D. Macdonald, J. Electrochem. Soc. 133 (1986) 2018.

[8] M. Urquidi-Macdonald, S. Real, D.D. Macdonald, Electrochim. Acta 35 (1990) 1559. 
[9] M.F.L. de Mele, D.A. Moreno, J.R. Ibars, H.A. Videla, Corrosion 47 (1) (1991) 24.

[10] N.B. De Cristofaro, C.A. Acosta, R.C. Salvarezza, H.A. Videla, Corrosion 42 (4) (1986) 241.

[11] B.A. Boukamp, Equivalent Circuit, Version 4.55, 1996.
Copyright B.A. Boukamp, University of Twente, 19931996.

[12] J.H. Wang, F.I. Wei, H.C. Shih, Corrosion 52 (8) (1996) 24.

[13] M. Drogowska, L. Bossard, H. Menard, A. Lasia, Mater. Sci. Forum 89 (1995) 192-194. 